\title{
Globe
}

Revue internationale d'études québécoises

\section{Jean-Christian Pleau : La révolution québécoise. Hubert Aquin et Gaston Miron au tournant des années soixante. Montréal, Fides, 2002}

\section{Martin Jalbert}

Volume 6, numéro 1, 2003

URI : https://id.erudit.org/iderudit/1000700ar

DOI : https://doi.org/10.7202/1000700ar

Aller au sommaire du numéro

Éditeur(s)

Globe, Revue internationale d'études québécoises

ISSN

1481-5869 (imprimé)

1923-8231 (numérique)

Découvrir la revue

Citer ce compte rendu

Jalbert, M. (2003). Compte rendu de [Jean-Christian Pleau : La révolution québécoise. Hubert Aquin et Gaston Miron au tournant des années soixante. Montréal, Fides, 2002]. Globe, 6(1), 176-180. https://doi.org/10.7202/1000700ar d'utilisation que vous pouvez consulter en ligne.

https://apropos.erudit.org/fr/usagers/politique-dutilisation/ 
possibles, faudrait-il du coup renoncer à la lutte de libération nationale? Assez curieusement, dans cette conclusion qui paraît en retrait par rapport aux analyses fort pertinentes qui la précèdent, tout se passe comme si l'auteur était tout à coup saisi lui-même par le malaise que son essai visait pourtant à dissiper! Comme quoi on ne se débarrasse pas facilement de la "mauvaise conscience " et de la culpabilité crucifiante qui l'accompagne lorsqu'on est franco-québécois d'héritage canadienfrançais; et il ne suffit manifestement pas de l'épingler pour en être débarrassé.

Jacques Pelletier

Université du Québec à Montréal

\section{Jean-Christian Pleau}

La révolution québécoise. Hubert Aquin

et Gaston Miron au tournant des années soixante.

Montréal, Fides, 2002.

Le paratexte annonce un ouvrage plutôt décapant, mû par le désir de relire "l'idée de "Révolution tranquille" "et de retrouver ce à quoi nous rend aveugles cette formule consacrée dont on ne saisit plus aujourd'hui " la valeur initiale de paradoxe ", à savoir le souhait, partagé par les intellectuels québécois des années 1960, d'une véritable révolution, dans ce qu'elle représentait de contraire à la tranquillité : "La Révolution tranquille, c'est en somme ce qui frappe de non-lieu la Révolution tout court " (p. 8). Ce n'est toutefois pas la pensée révolutionnaire à proprement parler qui occupe Jean-Christian Pleau, mais une partie de la "pensée politique " d'Hubert Aquin et de Gaston Miron, et plus particulièrement celle qu'élaborent l'essai "La fatigue culturelle du Canada français " et le poème "L'homme agonique ". Publiés en 1962 et en 1963 , ces deux " textes majeurs " (p. 11) sont choisis parce qu'ils correspondent au début de la " maturité " des deux écrivains et " au moment où la question de l'indépendance commen[ce] à occuper le premier plan de l'actualité " (p. 11). En deux chapitres, respectivement consacrés à Aquin et à Miron, l'étude se donne pour objets les influences intellectuelles et politiques des deux écrivains, les idées et leurs utilisations antérieures, l'inscription des textes dans leur contexte de rédaction et dans leur 
situation dialogique. Une partie du premier chapitre est consacrée à l'article de Pierre Elliott Trudeau, "La nouvelle trahison des clercs ", auquel répondait celui d'Aquin - les deux textes sont d'ailleurs reproduits dans le dernier quart du livre. Après avoir relevé notamment ce que la pensée antinationaliste de Trudeau doit à Dalberg-Acton, Pleau retrace les influences hétéroclites d'Aquin - a cette cobabitation de Barthes et de Teilhard[,] de Sartre et de saint Thomas d'Aquin * (p. 42) -, révélatrices, selon lui, de l'ancrage conceptuel et historique à la fois "traditionnel " et " contestataire " de l'intellectuel. L'essentiel du commentaire de l'essai aquinien porte sur les "généalogies d'idées ", c'est-à-dire sur le passé de certaines notions ou certains concepts qu'emprunte et recycle le texte, celles d'" écart " (C. Lévi-Strauss), de "culture globale " (E. B. Tylor, A. Césaire), de "métissage" (L. Senghor), de "fatigue culturelle" (A. Césaire, L. Kroeber), d' "enracinement "(M. Barrès). Cette étude des filiations a moins d'importance dans le chapitre consacré à Miron, où Pleau se concentre sur quelques images et mots-clés de "L'homme agonique ": les connotations politiques du "fou du roi ", les échos historiques de la "loi d'émeute ", la polysémie de l'" agonique " (comme lutte, joute et jeu), les diverses significations de la notion d'a aliénation . En contrepoint de la lecture du poème, Pleau aborde le Miron militant, son discours sur les rapports entre écriture et action politique, ainsi que les circonstances biographiques de son passage "d'un gauchisme purement social à l'indépendantisme militant * (p. 149).

Tout au long de l'ouvrage, l'analyse historienne se double d'une dimension ouvertement polémique qui contribue à inscrire la réflexion de Pleau dans le contexte idéologique actuel. Le retour à la politique des textes se veut explicitement une réaction contre la "désaffection généralisée à l'égard du politique "(p. 10), la réticence des littéraires à traiter de la question, l'indifférence des indépendantistes actuels à l'endroit de ces deux intellectuels et les mauvaises lectures qu'en font certains antinationalistes. Qui plus est, l'étude fait place à de multiples prises de position, parfois mordantes et efficaces, parfois rapides, largement investies de la subjectivité de l'essayiste, quelquefois envahissante mais plutôt attachante somme toute, d'autant qu'elle ne dissimule ni ses sympathies ni ses oppositions. Cette part proprement dialogique de l'étude est textuellement marquée par la présence d' apartés ", ces passages digressifs, en italique, qui confèrent à l'ouvrage un rythme intéressant. Lieux de discussions - sur l'antisémitisme de Lionel Groulx, le multiculturalisme anglophone, le ressentiment dont on accuse régulièrement le 
nationalisme et l'indépendantisme québécois - ou simples parenthèses (sur la manifestation de septembre 1963 lors de l'ouverture de la Place des Arts), ces apartés constituent la trace sensible de la nature double de l'ouvrage et de son ambivalence fondamentale: La révolution québécoise hésite entre deux formes, l'étude d'Aquin et de Miron et l'essai polémique dont le commentaire des ouvres semble fournir le prétexte. Il faut convenir que, de ce que disent les textes - dont il souligne pourtant que l'a argumentaire * et la " perspective [...] conservent toute leur pertinence " (p. 11) -, Pleau retient en définitive bien peu de choses. Ainsi at-on parfois l'impression que l'essai aquinien et le poème mironien sont convoqués pour la profondeur et la légitimité intellectuelles qu'ils confèrent à un discours qui les met peu à contribution, chose étonnante de la part de celui qui, contre l'indifférence des littéraires, soutient, en introduction, que les textes ont encore " quelque chose à nous apprendre du point de vue politique * (p. 10). L'ouvrage laisse plutôt penser que ce "quelque chose " ne nous concerne déjà plus. Cette impression provient en grande partie de la perspective historienne de l'analyse : s'il est certainement vrai que la connaissance de l'époque augmente souvent l'intelligence d'un texte littéraire, cette façon de faire de lui un "instantané historique * (p. 147), dont l'historien se propose pour nous de refaire le tirage, contribue moins à le rapprocher de nous qu'à le tenir à distance.

Élément principal de l'étude, l'examen généalogique des idées entraîne aussi des observations discutables. Par exemple :

Aquin ne paraissait pas même soupçonner la généalogie du concept d'enracinement : aucun livse de Barrès dans sa bibliothèque, aucune note à son sujet dans ses cahiers de lectures. Au demeurant, s'il avait eu de ce dernier ne fût-ce qu'une connaissance indirecte, peuton croire qu'il eût revendiqué l'enracinement avec si peu de souci de l'équivoque possible? (p. 77)

Il est incontestable que les mots charrient des connotations dont i] importe d'être conscient, mais à trop les enfermer dans leur ascendance et dans un réseau de déterminations duquel il devient difficile de les arracher, à établir ainsi des conditions d'admissibilité d'un discours (devant être orphelin de toute filiation suspecte), on risque non seulement de contribuer à rejeter de nombreux textes dans le silence, à les vider de leur charge potentiellement "révolutionnaire ", mais de substituer à la discussion d'idées à proprement parler une surveillance policière des 
sources. À deux endroits, Pleau remarque, au passage mais non sans une certaine pointe de mépris, que bien des gens se réclamant du marxisme n'avaient pas lu Marx: "On ne savait pas toujours au juste de quoi on parlait - (p. 154, voir aussi p. 80, note 40). Il importerait de déterminer davantage le référent de ce " on - qui, ici, discrédite plusieurs interlocuteurs valables, des militants d'abord mus par une conscience politique aux rédacteurs de Parti pris. Un tour de plus et ce devoir-avoir-lu, corrélatif de l'obligation de connaissance linguistique, conceptuelle et philosophique des mots qu'on emploie, se transforme en une pensée de l'orthodoxie. De cette conception généalogique des idées, découlent aussi les remarques récurrentes sur ces concepts et ces savoirs "de seconde main ", comme s'il existait un lieu originaire d'où les mots peuvent s'énoncer proprement. Des passages comme celui sur la notion d'، enracinement " ne contribuent certainement pas à démontrer en quoi les textes ont gardé de leur pertinence.

Non, ce n'est pas l'idée de révolution que Pleau retient des intellectuels des années 1960. Tout se passe au contraire comme si son désir de revenir à leur pensée politique et au rêve d'une "Révolution tout court . se heurtait à l'incapacité (la nôtre sans doute) de recevoir et d'assumer véritablement cette pensée et ce rêve : "Admettons-le, rien ne nous est plus étranger que l'appel à la Révolution " (p. 9). D'où cette étrange * Révolution québécoise ", qui ne descend pas de cet épigraphe si engageant, et dont il n'est en somme que vaguement question, comme si l'on ne savait qu'en faire. Les textes d'Aquin sur le sujet ne manquent pourtant pas - qu'on pense par exemple à Prochain épisode, à Trou de mémoire et à plusieurs de ses essais, "Le bonheur d'expression ", "L'existence politique - ou "Calcul différentiel de la contre-révolution. Chez Miron, en revanche, pas ou si peu de révolution, surtout pas au sens où l'entend Pleau de "rupture spectaculaire avec le passé - (p. 8) : " ici et là, le mot est prononcé, remarque justement Pleau, mais presque toujours furtivement et sans insister " (p. 171). Voilà qui souligne le gênant hiatus qu'il y a entre l'ouvrage et son paratexte.

La quatrième de couverture signale, non sans ambiguité, qu'a une relecture complète de l'idée de "Révolution tranquille" est à l'origine de ce double essai *. Nous ne saurons cependant rien, au terme de l'ouvrage qui a le mérite certain d'attirer notre attention sur la question, de ce que souhaitaient les intellectuels québécois comme "Révolution tout court . Quelle idée se trouve donc démontée et au profit de quelle autre? La 


\section{REVUE INTERNATIONALE D'ÉTUDES QUÉBÉCOISES}

photographie de la couverture nous fournit un élément de réponse : elle donne à voir une rangée de manifestants regroupés sous le mot d'ordre plus festif que contestataire - "vive la nation québécoise "; "quelques jeunes gens souriants, écrit Pleau lui-même, qu'on croirait être des collégiens surpris en plein chahut ; et quelques autres plus tranquilles, qui tâchent sans trop de succès de se donner un air menaçant " (p. 113, je souligne). Pourquoi donc chercher à décaper l'image d'une Révolution tranquille pour mettre à sa place celle d'une "Révolution québécoise " franchement juvénile, sympathique, souriante et dérisoirement menaçante? La "relecture * a-t-elle vraiment lieu? Non seulement l'ouvrage de Pleau reconduit-il le cliché d'une révolution on ne peut plus tranquille, mais il ne semble pas contredire, en définitive, le travail de deuil contemporain dont est l'objet la pensée révolutionnaire, voire la révolution tout court.

Martin Jalbert

Université Laval

\section{Paul Chamberland}

\section{En nouvelle barbarie. Essais}

Montréal, l'Hexagone, 1999.

Cette collection d'essais de Paul Chamberland s'inscrit - son titre l'annonce d'emblée - dans le vaste réseau de ces textes qui entendent diagnostiquer la société contemporaine : la "nouvelle barbarie * dont il est question se veut héritière de la "société du spectacle " de Guy Debord et de la "fabrique du consentement " de Chomsky et Herman (deux notions évoquées au passage, dans "Malaise généralisé *, p. 27). Elle affiche aussi un air de famille avec la *festivocratie" de Philippe Muray, l'" horreur économique " de Viviane Forrester, l' ère du vide * de Gilles Lipovetsky, la "défaite de la pensée "d'Alain Finkielkraut et le " devoir de bonheur " de Pascal Bruckner : autant de constats alarmistes (et parfois alarmants) sur l'état de notre civilisation fin-de-siècle. Par conséquent, le premier défi qui s'impose à l'auteur d'un tel ouvrage est d'éviter de prêcher pour les seuls convertis. Défi que Paul Chamberland ne relève que partiellement.

L'unité du recueil est assurée par la chronique éparse de "l'avancée du non-humain, ou de l'immonde (le non-monde) " ("Sujet citoyen ", 